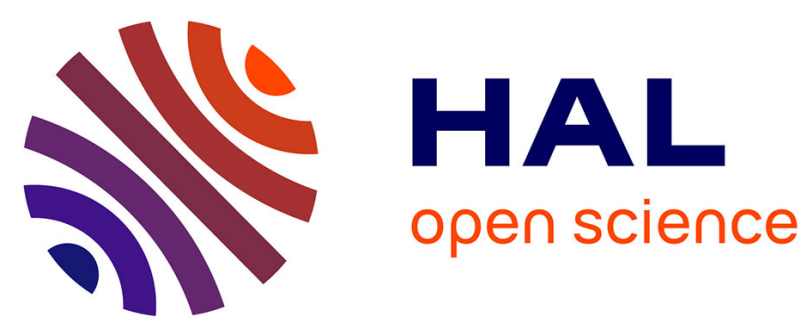

\title{
Cold compression of ceramic spray-dried granules: Role of the spatial distribution of the binder
}

Alice Boursier, Gaétan Grimaldi d'Esdra, Eric Lintingre, Christian Frétigny, François Lequeux, Laurence Talini

\section{- To cite this version:}

Alice Boursier, Gaétan Grimaldi d'Esdra, Eric Lintingre, Christian Frétigny, François Lequeux, et al. Cold compression of ceramic spray-dried granules: Role of the spatial distribution of the binder. Ceramics International, 2020. hal-03019864

\section{HAL Id: hal-03019864 https://hal.sorbonne-universite.fr/hal-03019864}

Submitted on 23 Nov 2020

HAL is a multi-disciplinary open access archive for the deposit and dissemination of scientific research documents, whether they are published or not. The documents may come from teaching and research institutions in France or abroad, or from public or private research centers.
L'archive ouverte pluridisciplinaire HAL, est destinée au dépôt et à la diffusion de documents scientifiques de niveau recherche, publiés ou non, émanant des établissements d'enseignement et de recherche français ou étrangers, des laboratoires publics ou privés. 


\title{
Cold compression of ceramic spray-dried granules: Role of the spatial
}

\section{distribution of the binder}

\author{
Alice Boursier ${ }^{\mathrm{a}, \mathrm{b}, \mathrm{c}}$, Gaétan Grimaldi d'Esdra $^{\mathrm{a}, \mathrm{b}}$, E. Lintingre $^{\mathrm{c}}$, Christian Frétigny $^{\mathrm{a}, \mathrm{b}}$, \\ François Lequeux ${ }^{\mathrm{a}, \mathrm{b}}$, Laurence Talini ${ }^{\mathrm{a}, \mathrm{b}, *}$ \\ a Sciences et Ingénierie de la Matière Molle, ESPCI Paris, CNRS, PSL University, \\ 75005 Paris, France \\ ${ }^{\mathrm{b}}$ Sorbonne Université, ESPCI Paris, Sciences et Ingénierie de la Matière Molle, 75005 \\ Paris, France \\ c Saint-Gobain Research Provence, 84306 Cavaillon, France \\ * corresponding author: laurence.talini@espci.fr Phone: +33 (0)140794679 \\ Postal address: Lab SIMM, ESPCI, 10 rue Vauquelin 75005 Paris
}

\begin{abstract}
We report an experimental study of the compaction behaviour of zirconia granules formed by a spray drying process. Using two different binders that were fully characterized, we have investigated the influence of the binder spatial distribution in the granules on compaction of a granule packing. Spatial distributions were modified by tuning binder adsorption on the ceramic nanoparticles in the spray dried suspensions. Granule powders with binder either located between nanoparticles only, or partly concentrated within a segregated layer at the surfaces of granules were thus obtained. We have found the role of the segregated layer is fully negligible during granule compression, and we justify that result by providing estimates of the theoretical compression energies of segregated layers and polymer bridges. We discuss the role of the binder bridges between nanoparticles, and we suggest a picture accounting for the
\end{abstract}


experimental influence of binder mechanical properties and concentration on the compaction behaviour of spray-dried granules.

Keywords: ceramics, binder layer, cold compaction, density, mechanical modelling

\section{Introduction}

It is well known that adding a polymeric binder to ceramic powders before their cold compression greatly improves the properties of the resulting green body. In particular, a binder increases the powder cohesion and thus the strength of the green body [1], opening the way for cold machining processes [2]. However, addition of a binder is also known to potentially result in a large porosity of the ceramics after the sintering step, during which all the organic content is removed. The optimum amount of binder is therefore determined by a compromise between the gain in green body strength and loss of density. In addition, the spatial distribution of binder in granules is as well known to have possible large consequences on the properties of the green body, but also of the sintered ceramics. It is well established that during spray drying process the binder can segregate to the surface of the granule, and further form a layer at that surface.

Evidences for that layer have been provided [3, 4] and it has been shown to form with binders that do not (or only poorly) adsorb onto the surfaces of the ceramic colloidal particle before spray drying. Segregated layers are suspected to have a role on green strength by enhancing the adhesion between granules $[1,5]$. However, they can be also detrimental to the quality of the final ceramics: their removal leaving coarse defects, segregated layers have been found to be at the origin of defaults in the final sintered ceramics [6]. They are also thought to have an effect on the structure of the compacts 
[4]. However further investigation is needed to understand the role of that layer during the compression step, for given mechanical properties of the binder.

In this work, we have used spray dried granules of zirconia to investigate the link between the compaction behaviour and the spatial distribution of the added binder. We have tuned the adsorption of the binder on the zirconia particles to determine the role of a segregated layer during compaction. The question we address is the following: what is the mechanical role of the binder and hence what should be the optimal distribution of a binder for the compaction step? The answer is supported by experimental data but also by a modelling of the behaviours at stake.

\section{Materials and methods}

\subsection{Zirconia granules}

Zirconia powders (Zirpro, Saint-Gobain) were de-agglomerated in water and milled with a repeatable water milling step to reach a median radius of $180 \mathrm{~nm}$ for the nanoparticles. During that step, dispersion of the nanoparticles was ensured by addition of either a dispersant (Dolapix Ce64 - Zschimmer \& Schwarz) or by increase of the zeta potential by $0.1 \mathrm{wt} \%$ acetic acid. The $\mathrm{pH}$ of the obtained suspensions (Zirconia volume fraction $15 \%$ ) was further modified in order to induce a slight aggregation of the Zirconia particles and promote the formation of spherical granules during the atomization process [7]. The suspensions were spray dried in a Niro atomizer after possible addition of a binder. Temperature of injected air was set at $280^{\circ} \mathrm{C}$, and outcoming air had a measured temperature of $110^{\circ} \mathrm{C}$. The spherical granules formed had an average diameter of $60 \mu \mathrm{m}$. The spray drying conditions were such that the Péclet 
number for the polymer was larger than 100 , ensuring that the polymer distribution is not sensitive to small variations of these conditions. As a result of the slightly aggregated state of the suspension, the granules are formed by a packing of colloidal aggregates comprising about ten particles. As shown, in the following, that specific structure is expected to influence granule compaction only at its very first stages.

\subsection{Polymers}

Two different binders were used: Poly(Vinyl Alcohol) (PVA) and an acrylic latex. They are listed in Table I together with their properties. Since PVA is plasticized by water, special care was taken to control the humidity rate in the granules. It was kept smaller than $0.5 \mathrm{wt} \%$. Glass transition temperatures of the polymers were measured by Differential Scanning Calorimetry (DSC) for the same humidity rate.

\subsection{Mechanical characterisation of polymers}

The polymers were dissolved in water, further lyophilised and molded at high temperature into parallelipedic samples of dimensions $1 \times 5 \times 20 \mathrm{~mm}$. Tensile tests were performed on the acrylic latex sample at an engineer extensional rate of $0.18 \mathrm{~s}^{-1}$ with a DMA Q800 (Dynamic Mechanical Analysis, TA Instruments), and on the PVA sample at a rate of $0.36 \mathrm{~s}^{-1}$ with a Zwick Roell.

Figure 1 shows the data correspond to true stress and strain measured for the different binders. The Young's modulus given in Table 1 are values averaged over 3 different samples for each polymer. As displayed in Fig. 1, the polymers exhibit qualitatively different mechanical behaviours at room temperature: PVA is semi-cristalline and 
below its glass transition and thus has a large modulus value and presents a fracture at a strain value of 5\%, while acrylic latex is viscoelastic and can sustain large strain values.

Plastic behaviour has been reported for PVA samples during tensile tests [8]. The measured yield stress was 55MPa and the yield strain $4 \%$, both values being very close to the stress and strain at break we have measured (Fig. 1). It is likely that defaults are at the origin of the rupture of our sample before occurrence of plastic deformation. In the following, we will consider PVA deforms plastically above the reported yield values.

\subsection{Polymer adsorption on Zirconia}

Adsorption of polymers on Zirconia was determined by measuring the equilibrium concentration of polymers in the interstitial solution using Total Organic Carbon method (TOC-VCSH, Shimadzu). The amounts of polymer adsorbed on nanoparticles were inferred from these measurements and they are shown in Fig. 2 as a function of the initial polymer concentration in the suspensions. Clearly, the adsorbed amount of PVA plateaus at $0.5 \mathrm{wt} \%$ whereas acrylic latex strongly adsorbs onto Zirconia and no plateau was observed within the measured range. However, Fig. 2 shows that addition of a dispersant almost fully inhibits the adsorption of acrylic latex. It is therefore possible to obtain samples in which acrylic latex adsorbs either fully or only very poorly onto the nanoparticles. Since a segregated polymer layer is expected at the surface of spray dried granules from samples in which the polymer is free, in the following, we tune the binder adsorption with dispersant in order to investigate the role of polymer segregated layers on the compression of green bodies. 
In addition, we have used the thermal treatment suggested in [3] in order to visualize the segregated polymer layers at the surface of spray dried granules. Such layers were evidenced in samples contaning both an adsorbing polymer (Acrylic latex or PVA) and a dispersant to inhibit polymer adorption on Zirconia. In contrast, a sample made with acrylic latex and no dispersant did not exhibit these layers after thermal treatment, confirming that no segregated layer forms with a fully adsorbing polymer.

The thickness of the formed segregated layers can be estimated from the polymer content, that point will be addressed in the following.

\subsection{Compaction experiments}

A given weight $(0.105 \mathrm{~g})$ of granule powder was poured in a cell of diameter $5 \mathrm{~mm}$. Compaction experiments were carried out using a Zwick Roell equipped with a $10 \mathrm{kN}$ force sensor. After a pre-compression at $0.04 \mathrm{MPa}$ in order to set the zero-displacement position, the sample was compacted at a constant rate of $0.02 \mathrm{~mm} \cdot \mathrm{s}^{-1}$. All compactions were performed at $20^{\circ} \mathrm{C}$.

\section{Results}

\subsection{Effect of binder segregated layers on the compaction behaviour}

In Fig. 4, we compare the compaction behaviour of granules made with the two different binders, with and without addition of dispersant for each binder. In agreement with reports in the literature, the compaction is a two-step process in all cases: first, the volume fraction only weakly varies with pressure, but above a threshold pressure, 
density increases linearly with the logarithm of the pressure. The curves quantitatively differ according to the nature of the binder, which is expected considering the strongly different mechanical properties of the binders. In particular, the threshold pressure is larger for PVA than for acrylic latex. It however varies by less than one decade between both polymers whereas the Young's modulus of the two binders differ by three orders of magnitude. Finally, at pressures larger than ca. 10MPa, all curves converge to the same limit curve. Therefore, the nature of the binder influences the compaction process only within a limited pressure range.

We now focus on the role on compaction of the binder distribution in the granules. As detailed above, acrylic latex strongly adsorbs onto the surfaces of the nanoparticles in the absence of dispersant and only very poorly in the presence of dispersant. Therefore, the curves obtained with and without dispersant respectively correspond to granules having a binder layer at their surfaces (dashed line) or not (full line). As a reference, in Fig. 4 we show the curves obtained with and without dispersant with a PVA binder that poorly adsorbs onto the surface of the nanoparticle. Only $0.5 \%$ wt is expected to adsorb, which represents $25 \%$ of the total binder content. A segregated layer therefore forms with and without dispersant, its thickness being smaller by $25 \%$ without dispersant (full line) than with dispersant (dashed line).

For acrylic latex, the threshold pressure is smaller by about $20 \%$ when a segregated layer is present. A similar feature was reported during the compaction of alumina with a polyacrylic acid binder [5]. However, in the present experiments, the differences of behaviour of the samples in which the binder distribution has been tuned remain very close to the ones of the reference samples in which segregation layers are present. It is therefore likely that the differences we observe result from dispersant addition. We can 
therefore conclude from the data of Fig. 4 that tuning the binder distribution does not result in any significant modification of the compaction curves.

The influence of segregated layers has been examined in past studies. In particular, the compression behaviour and structure of the formed compacts were investigated in alumina with two different binders: PVA that forms a segregated layer during spray drying, and poly-acrylic acid (PAA) that is homogeneously distributed within the granules [4]. Differences in the compression curves and structures were observed. The internal structure of the granule was also found to differ with the used binders. However, the binder distribution was not varied independently of its mechanical properties. PVA and PAA actually differ by both their Tg and their state, PVA being semi-crystalline and PAA amorphous. We therefore suggest that the differences reported in Tanaka et al.'s paper mainly originate from the binder mechanical properties rather than from the distribution of the binder. In the present work we demonstrate that, whereas the compaction behaviour clearly depends on the mechanical properties of the binder, tuning the formation of a segregated layer does not significantly modify the compaction curves for a given binder. We show in the following that this result is consistent with the negligible contribution of the binder layer to the compaction energy. In addition, we show that the main contribution to that energy results from the deformation of the polymer between nanoparticles. In the next paragraph, we detail how, even when the binder does not adsorb onto the surfaces of nanoparticles, part of it is expected to remain between nanoparticles within the granules.

\subsection{Binder distribution}


The presence of binder between nanoparticles results from binder adsorption at the surface of nanoparticles but part of the binder can also be trapped if it does not adsorb and remains free in the solvent. Actually, during the high Peclet numbers drying involved in spray-drying, part of the free binder concentrates at the surface of the granules to form the segregated layer, but another part remains trapped in capillary bridges between nanoparticles. An estimate of the amount of binder trapped between nanoparticles can be provided by considering the drying process of a porous medium [9]. The stage of drying at which the nanoparticles reach the random close packing and the air/solvent interface penetrates in the granules is the funicular stage. During that stage, there are continuous solvent paths in the granule and the solvent keeps concentrating at their surface. However, as drying proceeds, the paths become discontinuous. During the final drying regime (pendular regime), the solvent is trapped within capillary bridges between the surfaces of neighbouring nanoparticles. As a consequence, once drying is completed, roughly $15 \%$ of the binder is located between neighbouring nanoparticles [9] if the binder is initially free in the solvent, as already pointed out in the literature [10].

More quantitatively, estimates of the thicknesses of the segregated layer and of the binder bridges can be provided for given binder content and adsorption. Assuming homogeneous repartition of the binder either around the granules or the Zirconia particles yield simple geometric relations giving the thicknesses as a function of the volume fractions of the adsorbed and free binder. The results are summarized in Table II.

The thickness of the segregated layer for poorly adsorbing polymer is a fraction of micron, in agreement with results from the literature [10]. The thickness of the bridges 
between nanoparticles are of the same order of magnitude for both binders. The latter values are only indicative averages since they do not account for the heterogeneous distribution of the polymer that is expected around the nanoparticles.

In conclusion, if the binder does not adsorb on the nanoparticles surface, at the end of the spray drying process, about $85 \%$ of its initial content is distributed in segregated layers at the surface of the granules and the remaining part is trapped in bridges between nanoparticles surfaces. Therefore, a significant amount of binder is expected to remain between nanoparticles, whatever their interaction with the binder. In the following, we compare the effects of the deformation of segregated layers and polymer bridges during compaction.

\subsection{Discussion: Modelling the role of the binder}

In Appendix A, we provide an estimate of contribution of the binder layer to compaction of ceramic granules. We show that the folding energy of the segregated layer has three contributions. Actually, when layers are packed, their shapes evolve towards a polyhedral shape and the transformation from a spherical shell to a polyhedron implies formation of faces, as well as edges and vertices. In appendix A, we show that the binder layer at the vertices opposes the largest resistance to compaction. That effect can be quantified by the void volume fraction at the vertices, which is shown as a function of pressure in Fig. 5. It is compared to the total void volume fraction inferred from the compaction curves of Fig. 4.

Figure 5 demonstrates that the voids created by the deformation of granules into polyhedrons are filled by the binder layers at an early stage of the compaction process. 
For instance, at pressure values larger than $0.2 \mathrm{MPa}$, the segregated layers do not oppose any resistance to compaction even when they are constituted by a large Young modulus binder as PVA.

As a result, the contribution of the binder to the mechanical behaviour during compaction mainly originates from the binder bridges between nanoparticles. As pressure increases, the bridges are expected to respond differently according to the binder's nature. As shown by mechanical characterisation, acrylic latex deforms elastically up to large strains. In contrast, PVA deforms plastically. In the approximation of an isotropic pressure equally distributed over all the bridges, we derive the relation between the macroscopic pressure and the thickness $h$ of the bridge in Appendix B. For an elastic deformation, and a binder volume fraction larger than $0.6 \%$, the macroscopic pressure is independent of the binder volume fraction and is simply given by:

$P \approx \frac{E}{2}\left(1-\frac{h}{h_{0}}\right)$

This relation is derived in Appendix B. It demonstrates that at pressure values close to the Young's modulus of the binder, the gap between particles goes to zero, and friction between nanoparticles starts influencing compaction. Remarkably, the threshold depends on the polymer content only through the initial thickness of the bridges, $h_{0}$. At a given pressure value, the thickness of polymer bridges is therefore all the more larger than the initial thickness is large.

In the case of a plastic deformation the pressure at yield point is shown in Appendix B to be: 
$P=\sigma_{y} \frac{2}{3}\left(\frac{\varphi}{3 Z f_{c}}\right)^{1 / 4} J\left(\left(\frac{\varphi}{3 Z f_{c}}\right)^{1 / 4} \sqrt{\frac{R_{p}}{h_{0}}}\right)$

Where $\sigma_{Y}$ is the yield stress, $\varphi$ the volume fraction of polymer distributed in the bridges, $Z$ the number of half-capillary bridges by nanoparticle, and $f_{c}$ the volume fraction of solid particles. $J$ is the function defined in appendix B. $R_{p}$ and $h_{0}$ are respectively the radius of a nanoparticle and the thickness of the capillary bridge. Typical values for a disordered packing of spheres were used to provide numerical estimates: $Z=6$ and $f_{\mathrm{c}}=0.6$. The volume fraction of polymer in the bridge is expected to be the same as the total volume fraction for a fully adsorbing binder, and only $15 \%$ of that content for a non-adsorbing one.

Below this pressure threshold, the binder bridge does not yield and respond elastically. At pressures larger than the threshold, the bridge thins down until its thickness reaches the value corresponding to the applied pressure. As shown in fig. 6 , the yielding pressure is always significantly smaller than the polymer yield stress. For bridges of thickness $h_{0}=6 \mathrm{~nm}$, the yielding pressure is close to $0.1 \sigma_{y}$. In addition, according to eq. (2), the yielding pressure only weakly depends on the polymer volume fraction, which in agreement with the weak variation of the compaction curves observed by adding dispersant (thus changing the content of polymer in the bridges).

In the above description, we have assumed a homogeneous distribution of the binder in the granules. In the case of a non adsorbing binder, concentration gradients are actually expected in the granules. However, the curves of Fig. 4 show that the differences between a fully adsorbing binder (for which the distribution is homogeneous) and a non-adsorbing binder are very small. In addition, as already mentioned, we have found 
the dependence on binder volume fraction is weak, which further justifies the assumption of a homogeneous distribution.

Equations (1) and (2) provides estimates for the threshold pressures above which friction between nanoparticles start opposing compaction. The values for Acrylic latex and PVA are indicated in Fig. 5 (dashed lines) and they roughly correspond to the change in the slope of the compaction curves. Owing to the heterogeneous distribution of polymer between nanoparticles, a wide distribution of bridge thicknesses is expected, and above the threshold pressures polymer bridges still influence compaction. Actually, the compaction curves are observed to tend toward a limit curve at much larger pressure values (close to $100 \mathrm{MPa}$ ), which is indicative of a compaction driven by friction only at that stage.

In summary, we suggest the following picture: During drying, the binder may concentrate at two scales, forming layers at the surface of granules and capillary bridges between neighbouring nanoparticles. The segregated layer only forms for a nonadsorbing binder, whereas the capillary bridges form even in the case of a nonadsorbing polymer owing to solvent trapping during drying. During granule compaction, the segregated layer contribution to pressure is fully negligible. The voids at the scale of the granules are only maintained because of the binder forming bridges between nanoparticles that significantly oppose compaction. However, when the pressure is increased, the voids begin to disappear because the bridges deform significantly. This corresponds also to the pressure at which the bridges collapse and solid friction starts opposing compaction. Last, the threshold pressure only weakly depends on the binder volume fraction. As a result, compaction only weakly depends on the nature of the binder and its volume fraction. The onset of compaction regime is just 
set by the collapse of the inter-particle bridges. Compaction is driven by a coexistence between the increasing number of solid/solid friction in between particles, and the decreasing number of remaining binder bridges.

\section{Conclusion}

In conclusion, we have investigated the role of the segregated binder layer on compaction behaviour of spray dried zirconia granules. We have used a dispersant to tune the formation of the layer for the same (Acrylic latex) binder. We demonstrate segregated layers have no significant influence on compaction, which is fully explained by a model showing the layers deform during the early stages of compaction. In contrast, the compaction curves depend on the mechanical properties of the binder bridges between nanoparticles. We explain that result by demonstrating compaction is driven by compression of the binder bridges between nanoparticles, that may collapse. This collapse results in particles/particles solid friction, and we provide an analytical expression for the resulting macroscopic pressures of the onset of friction, that are in agreement with our observations. Our analysis opens the way for a better understanding of the influence of the nature of the binder on compaction of spray-dried granules, and in particular on the relevant mechanical properties of the binder. In addition, it shows the optimal distribution of the binder should be determined by considering other steps of ceramic fabrication process than cold compaction.

Acknowledgements: The study was funded by ANRT and Saint-Gobain Research Provence. The authors thank Hélène Montes for fruitful discussions on the mechanics of polymeric materials. 


\section{Appendix A}

\section{Mechanics of the segregated layer around granules}

We aim at providing estimates of the contribution of an elastic shell on granule compression. In order to isolate the contribution of the shell, we first assume that there is no cohesion between nano-particles. That rough approximation allowing estimates of the contribution of the shell mechanics on the compaction. In that case, as usually done in granular matter we assume that the stress is isotropic. We estimate the elastic energy corresponding to the deformation of the shells under compaction, which transforms the granule from a spherical shape to some polyhedral one as schematized in figure A1.

We will show that the shell contribution is fully negligible, as a result and for the sake of simplicity, we provide a rough estimate of the shell contribution to. In the first stage of compression, due to the packing of the granules, the parts or the granules surfaces that are around the initial contact points flatten, as depicted in figure A.2. The granules progressively flatten against their neighbours at the middle of the faces of the polyhedra. As pressure increases, these faces tend towards the ones of the final polyhedra. The last stage of compression involves two mechanisms, namely folding at the edges and folding at the vertices.

Let us first provide estimates of the contribution of the flattening of the granules one against the others. The elastic energy of the shell increases while granules flatten on the face of the polyhedra, due to the change of curvature of the shell.

First we estimate the volume of the void. The volume of a conical part (with an angle $\beta$ ) of a truncated sphere of radius $\mathrm{R}_{g}$, with a truncation of $h$ with respect to the apex) is: 


$$
\mathrm{V}_{t r}=\frac{1}{3} \pi\left(-3 h^{2} \mathrm{R}_{g}+2 \mathrm{R}_{g}{ }^{3}-2 \mathrm{R}_{g}{ }^{3} \operatorname{Cos}(\beta)\right)
$$

When the granules are compressed, an amount of matter is displaced from the truncation. As a result the sphere increases its radius, by a quantity $\delta_{t r}=\frac{h^{2}}{2 \operatorname{Rg}(\operatorname{Cos}(\beta)-1)}$, which is of the second order in $h$ and that will be neglected in the following.

The void is just the difference between the pyramid with a height of $R_{g}-h$ and the sphere, on the considered cone. Its values is, up to the first order in $h$, is given by:

$$
V=\frac{1}{3} \mathrm{R}_{g}{ }^{3}\left(2 \pi(\operatorname{Cos}(\beta)-1)+\pi \operatorname{Tan}(\beta)^{2}\right)-\pi \mathrm{R}_{g}{ }^{2} \operatorname{Tan}(\beta)^{2} h
$$

Therefore:

$$
\frac{\partial V}{\partial h}=-\pi \mathrm{R}_{g}^{2} \tan (\beta)^{2}
$$

We have now to provide an estimate the elastic energy induced by the flattening of the spherical shell. It combines bending and dilation, but bending is negligible compared to dilation [11]. However, the dilation is a compromise between radial and orthoradial deformation which are expected to be of the same orders of magnitude. Our aim here is not to solve exactly that very difficult problem but rather to show that the shell contribution to the pressure can be neglected. We will thus overestimate the elastic energy, by a factor about 2 , by assuming the orthoradial deformation is zero. To that extent, we consider the following deformation, which clearly overestimate the energy by assuming that the orthoradial stress remains zero. A circle of matter is initially located on the sphere and centred on the cone axis, has an initial radius $\mathrm{R}_{g} \sin \varphi$ where $\varphi$ is the polar angle indicated on figure A.2. It is displaced to the flat surface keeping a 
constant radius, its centre remaining on the cone axis. The radial deformation is just simply given by $(\cos (\varphi)-1)$. The energy is therefore given by:

$$
U_{F}=\frac{E e}{2} \int_{0}^{\omega}(\cos (\varphi)-1)^{2} 2 \pi R_{g}^{2} d \varphi
$$

The integration starts from the apex $-\varphi=0$ to an angle $\varphi=\omega$. This last angle is given by the the value at which the sphere of radius $\mathrm{R}_{g}$ intersects the face, at $\mathrm{z}=\mathrm{R}_{\mathrm{g}}-\mathrm{h}$. This writes : $\cos (\omega)=1-\frac{h}{R g}$. After integration, up to the lowest order in $h$, we get :

$$
U_{F}=\frac{\sqrt{2} \mathrm{E} e \pi h^{5 / 2}}{5 \mathrm{R}_{g}^{3 / 2}}
$$

To obtain the pressure, we use the classical relation $P=\frac{-\partial U_{F}}{\partial V_{t r}}$. With $\beta=\pi / 6$, it yields:

$$
P=\frac{18 \sqrt{2} \mathrm{Eeh}^{3 / 2}}{\operatorname{Rg}^{5 / 2}}
$$

This pressure contribution of the face varies from zero to about $25.5 \mathrm{Ee} / \mathrm{R}_{\mathrm{g}}$ (for $h$ varying from zero to $\mathrm{R}_{\mathrm{g}}$ ), and we will see above that this contribution is small when compared to the one of the vertices and the edges.

When the flattening of the shells becomes important, the mechanical energy concentrates into the folding at the edges and the curvatures near the vertices of the polyhedra. Estimates of the pressure must therefore be given in these two situations.

We assume in the following that contributions of the folding at edges and vertices are independent, they have their own voids and folding energy. The pressure is estimated as the derivative of the energy versus the voids density for each situation. We will see at 
the end that the flattening of the granules at the faces is negligible as compared to the folding at the edges and the vertices. We first consider the edges.

During the final stage of compression, around each edge, the surface withdraws from the polyhedra. This defines the voids regions near the edges and the vertices. For the sake of simplicity, we assume that the edge lengths are constant, their values being nearly equal to $R_{\mathrm{g}}$ and that there are $N_{E}$ edges per granule. We also assume that the edges are symmetrically shared between three adjacent granules. A section perpendicular to the edge is shown in fig. A.3.

The area of the void region, associated to one granule, in a plane perpendicular to the edge is $(\sqrt{3} / 3-\pi / 6) l_{E}^{2}$. The volume of the void domain along the edges, per granule, is then about $V_{e} \simeq 0.054 N_{E} R_{g} l_{E}^{2}$, where $N_{E}$ is the number of edges per granules, and taking $\mathrm{R}_{\mathrm{g}}$ for the length of the edges. Using the classical theory of shells [12], one can show that for this bending mode, the shell of thickness $e$ and of modulus $E$, has an energy per surface unit given by $E e^{3} \frac{\left(l_{E}-R_{g}\right)^{2}}{24 l_{E}^{2} R_{g}^{2}}$. In the limit of $l_{E}$ small compared to $R_{\mathrm{g}}$, it reduces to $\frac{E e^{3}}{24 l_{E}^{2}}$. Per granule, the contribution has thus to be multiplied by the length of the arc, $\frac{\pi}{3} l_{E}$ and by the total length of the edges of the granule, $N_{E} R_{g}$. This leads to a total energy, per granule for the folding at the vertices given by:

$$
U_{e} \simeq \frac{\pi}{72} N_{E} E \frac{e^{3} R_{g}}{l_{E}}
$$

The contribution to the pressure can now be deduced. It is given by the derivative of the energy with respect to the volume. Note that the pressure is independent of the number of edges and writes 


$$
P_{e}=\frac{-\partial U_{e}}{\partial l_{E}} \frac{\partial l_{E}}{\partial V_{e}}
$$

Using the above equations, one gets

$$
P_{e} \approx 0.41 E\left(\frac{e}{l_{E}}\right)^{3}
$$

We can now writes the pressure as a function of the void - at edges - volume fraction $\varphi_{e} \simeq 0.053 N_{V} R_{g} l_{E}^{2} /\left(\frac{4}{3}\right) \pi R_{p}{ }^{3}$, for the contribution of the edges:

$$
P_{e} \approx 0.097 E \frac{e^{3}}{\varphi_{e}^{3 / 2} R_{g}{ }^{3}}
$$

We can now consider the case of plastic shell. Let us here assume that the yield stress is reached within the curved shell. The deformation remains elastic near the middle of the shell, between $-\mathrm{ey}$ and $+\mathrm{ey}$, and writes thus $\frac{z}{l_{E}}$ between $-\mathrm{ey}$ and $+\mathrm{ey}$. Outside this domain, the deformation is plastic and takes the values of $\pm \varepsilon$ y on respectively outer and inner parts of the shell. The value of ey is given by $e_{Y}=\varepsilon_{Y} . l_{E}$. The energy writes : $\varepsilon_{Y}^{2}\left(e-\frac{2}{3} \varepsilon_{Y} \cdot l_{E}\right)$. This yields for the energy per granules to $\varepsilon_{Y}^{2}\left(e-\frac{2}{3} \varepsilon_{Y} \cdot l_{E}\right) N_{V} R_{g} \frac{\pi}{3} l_{E}$. This energy is an increasing function of $l_{E}$ when $l_{E}$ is smaller than $\frac{3 \mathrm{e}}{4 \varepsilon_{Y}}$. This leads to negative pressure, or an instable condition and $l_{E}$ tends towards zero. Thus when the yield stress is reached in the shell, it spontaneously folds into the vertex wedge.

The folding at the vertices is more delicate to estimate. As explained above, the dilation is a compromise between radial and orthoradial deformation. As done for flattening, we assume a zero orthoradial deformation. To account for the deformation of the shell near the vertices, we assume that the vertices can be replaced by a right circular cone of angle $\alpha \sim \pi / 3$. The spherical surface of the shell is tangent to the cone at the location 
where the cone and the granule are in contact. The shell is assumed to be spherical with its initial curvature close to the apex and conical on the other side of the contact line. Let us consider the following deformation, that clearly overestimate the energy by assuming that the orthoradial stress remains zero. A circle of matter is initially located on the sphere and centred on the cone axis, has an initial radius $\operatorname{Rg}_{\mathrm{g}} \sin \varphi$ where $\varphi$ is the polar angle indicated on figure A4. It is displaced to the cone surface, with a constant radius, its center being kept on the cone axis. This deformation gives an upper bound for the actual elastic energy of the deformation.

The elastic energy is related to the extension of the shell along the cone direction. More precisely if two circles are distant of $\mathrm{Rg}_{\mathrm{g}} \varphi$, before deformation, their distance will become, after deformation:

$$
R_{g} \frac{\cos (\varphi)}{\sin (\alpha)} d \varphi
$$

The shell is assumed to be elongated only in the direction of the cone axis, and its elongation will be:

$$
\varepsilon=\frac{\cos (\varphi)}{\sin (\alpha)}-1
$$

Note that the maximum of deformation is reach at the vertices and is $\frac{1}{\sin (\alpha)}-1$. Taking $\alpha=\pi / 3$ this maximum strain is about 0.15 , just above the typical value of the yield strain of glassy or semi-crystalline polymer, 0.05 . In this case of yielding polymer, one must just replace $\frac{\cos (\varphi)}{\sin (\alpha)}-1$ by the yield strain in the following formulas to have a good order of magnitude. Thus the elastic energy required to push the elastic shell against the cone is: 


$$
U_{b}=\frac{E e}{2} \int_{\psi}^{\pi / 2-\alpha}\left(\frac{\cos (\varphi)}{\sin (\alpha)}-1\right)^{2} 2 \pi R_{g}^{2} d \varphi
$$

The distance at which the shell comes away from a face to the vertex $l_{V}$, requires that the integration boundary is set by $\sin \psi \simeq \frac{l_{V}}{2 R_{g}}$.

The void volume can be estimated as the difference between the one of the portion of the cone and that of the spherical cap (see figure A.4). The volume of the cone writes, in between the contact line:

$$
V_{\text {cone }}=\frac{l_{V}^{3} \pi}{3} \cdot \sin ^{2} \alpha \cdot \cos \alpha
$$

The one of the truncation of the granule writes:

$$
V_{c a l}=\pi R_{g}^{3}\left(1-\cos \beta+\frac{\cos ^{3} \beta-1}{3}\right)
$$

We have also in the triangle AOC the geometrical relation:

$$
\frac{R_{g}}{\sin \alpha}=\frac{l_{V}}{\sin \beta}
$$

Assuming that $l_{V}$ is smaller than $\mathrm{R}_{\mathrm{g}}$, we obtain the void volume :

$$
V_{\text {cone }}-V_{\text {cal }}=\pi \cos \alpha \sin ^{3} \alpha l_{V}^{3}
$$

For $\alpha=\pi / 3$, this writes $V_{\text {cone }}-V_{\text {cal }}=1.02 l_{V}{ }^{3}$

Using the relation between pressure, the energy and the volume and $l_{V}$ allows to estimate the pressure $P_{b}=\frac{-\partial U_{b}}{\partial V}$. It yields: 


$$
P_{b} \cong 4 E \frac{e R_{g}}{l_{V}^{2}} \tan (\alpha)\left(\frac{1}{\sin (\alpha)}-1\right)^{2}
$$

The volume fraction of voids is:

$$
\varphi_{V} \simeq \frac{1.02 l_{V}^{3} N_{V}}{\frac{4}{3} \pi R_{g}^{3}}
$$

Eliminating $l_{V}$ between the two previous relation leads to the relation between the vertex contribution of pressure. With $\mathrm{N}_{\mathrm{v}}=20$ and $\alpha=\pi / 3$, one gets:

$$
P_{b} \cong E \frac{0.48 \mathrm{e}}{\varphi_{V}^{2 / 3} R_{g}}
$$

In the case of yielding polymer, the relation can be obtained just replacing $\left(\frac{1}{\sin (\alpha)}-1\right)^{2}$ by $\varepsilon_{Y}^{2}$ :

$$
P_{b} \cong E \frac{20 \mathrm{e} \varepsilon_{Y}^{2}}{\varphi_{V}^{2 / 3} R_{g}}
$$

\section{Appendix B}

\section{Mechanics of the binder bridges}

The role of elastic bridges in granular materials has been investigated in [13]. The macroscopic elastic modulus was observed to be about ten times the one of the elastic bridges. This should have important consequences on the elastic recovery. The stress $\sigma$ stored in the elastic bridges during compression, after load cessation, leads to a recovery strain of about one tenth of $\sigma / \mathrm{E}$. The observed recovery above $10 \mathrm{MPa}$ is about $\varepsilon_{R}=4 \%$. For the acrylic binder, it corresponds to a stress stored in the elastic bridges of 
the order of $10 E \varepsilon_{r}=0.4 M P a$. AS a result, the elastic stress stored in the polymer bridges between nanoparticles has a negligible contribution to the macroscopic strain. Most of the strain is thus sustained by the granular network. We will now provide estimates of the thickness of the gap between neighbouring particles under stress.

In a first stage, we consider two neighbouring nanoparticles of radius $R_{p}$. The distance between the particle surfaces is $\mathrm{h}_{0}$. The distance between the two particles surfaces depends thus on the distance $r$ between their common axis - as shown in figure B1. This distance is given by, at the lowest order in $r / R_{p}$ :

$$
h(r)=h_{0}+\frac{r^{2}}{R_{p}}
$$

We denote as $a$ the radius of the binder bridge between the particles. The volume of binder $v$ in a bridge is thus:

$$
v=\int_{0}^{a} h(r) 2 \pi r d r=h_{0} \pi a^{2}+\frac{\pi a^{4}}{2 R_{p}}
$$

It is convenient to write the relation between the volume fraction $\phi$ of binders involved in the capillary bridges and $a$. Assuming $\mathrm{Z}$ half-capillary bridges by particle, and a volume fraction of solid particles of $f_{C}$, we have the relation $v=8 \pi R_{p}^{3} \varphi / 3 Z f_{c}$. Assuming $h_{0}<<\mathrm{R}_{\mathrm{p}}$, it is easily inferred from (B.2) that:

$$
a=2 R_{p}\left(\left(\frac{\varphi}{3 Z f_{c}}\right)^{1 / 4}+O\left(\frac{h_{0}}{R_{p}}\right)\right)
$$

The stiffness of a pair of particles, submitted to a displacement $\delta$ parallel to the axis of symmetry in the absence of wall slipping can be estimated. We assume that the binder is 
incompressible, and we use a lubrication approximation as usually performed in thin films hydrodynamics. We make the hypothesis that the distance between the two particles decreases from $h_{0}$ to $h_{0}-\delta$. In the frame of a lubrication approximation, the displacement profile between the two particles is parabolic. In consequence, the radial displacement has the following shape:

$$
u_{r}=A z(h-z)
$$

Where $A$ is a function of $r$ only. This profile assumes that there is no slip at each of the binder-particles interface. The incompressibility requires that, for each cylinder of radius $r$ centred around the axis of symmetry, the decrease of volume due to the displacement $\delta$ is exactly compensated by the volume of binder that goes out of the cylinder. This writes:

$$
2 \pi r \int_{0}^{h} u_{r} d z+\pi r^{2} \delta=0
$$

This determines $\mathrm{A}$ and gives $\mathrm{A}=-3 \mathrm{r} \delta / \mathrm{h}^{3}$.

If the binder is elastic, the energy of a compressed bridge is given by:

$$
W_{e l}=\frac{G}{2} \int_{0}^{a} 2 \pi r d r \int_{0}^{h(r)}\left(\frac{\partial u_{r}}{\partial z}\right)^{2} d z
$$

Which yields, using eq. (B.4) and (B.3):

$$
W_{e l}=\frac{3 G \pi R_{p}^{2} \delta^{2}}{4 \mathrm{~h}_{0}}\left(1+O\left(\frac{2 \sqrt{3} h_{0} Z f_{c}}{R_{p} \varphi^{1 / 2}}\right)\right)
$$

which at the lowest order in $\mathrm{h}_{0}$ is independent of the volume fraction, provided $\varphi>$ $\sqrt{\frac{2 \sqrt{3} h_{0} Z f_{c}}{R_{p}}} \approx 6.10^{-3}$ using the same parameters than below. So apart for extremely low volume factions of binder, the force of the sandwich particle/binder bridge/particule is 
equal to : $\frac{3 G \pi R_{p}^{2} \delta}{2 \mathrm{~h}_{0}}$, and thus a relation between the macroscopic pressure and the gap is given by :

$$
h=h_{0}\left(1-\frac{2 P}{3 G}\right)
$$

Note that the maximum of deformation is about $\sqrt{R_{p} / h_{0}}$. This deformation can easily be overcome for glassy or semi-crytalline polymer for which the yield strain is about $5 \%$. The previous equation is valid as long as the volume fraction is large enough as can be seen in eq. (B.7). In that case, local mechanical equilibrium yields the relation:

$$
-\frac{\partial P}{\partial r}+\frac{\partial \sigma_{r z}}{\partial z}=0
$$

Eq. (B.9) provides the magnitude of the pressure gradient.

$$
\frac{\partial P}{\partial r} \approx-\frac{\sigma_{y}}{h}
$$

The pressure is thus the integral of the previous expression:

$$
P(r)=\int_{r}^{a} \frac{\sigma_{y}}{h_{0}+\frac{r^{\prime 2}}{R_{p}}} d r^{\prime}
$$

Which gives after integration:

$$
P(r)=\frac{R_{p} \sigma_{y}\left(\operatorname{Arctan}\left(\frac{a}{\sqrt{h_{0} R_{p}}}\right)-\operatorname{Arctan}\left(\frac{r}{\sqrt{h_{0} R_{p}}}\right)\right)}{\sqrt{h_{0} R_{p}}}
$$

As a result, the force between the particles, which corresponds to the integral of the pressure over the bridge section is:

$$
F=\pi R_{p} a \sigma_{y}\left(1-\frac{\sqrt{h_{0} R_{p}}}{a} \operatorname{Arctan}\left(\frac{a}{\sqrt{h_{0} R_{p}}}\right)\right)
$$


Which corresponds to a macroscopic pressure of $\mathrm{F} / \pi \mathrm{RP}^{2}$.

In consequence, the relation between the pressure and the bridge height yields:

$$
P=\frac{a}{R_{p}} \sigma_{y} J\left(\frac{a}{\sqrt{h_{0} R_{p}}}\right)
$$

We note $J(x)=1-\operatorname{Arctan}(x) / x$. The series expansion of $J$ is $J(x)=\frac{x^{2}}{3}+O\left(x^{4}\right)$ and $J(x)$ tends towards 1 for $\mathrm{x}$ going to infinity. We can replace the value of the bridge radius by eq. (B.3) and thus obtain the relation between the macroscopic pressure and the thickness of the binder bridge at the onset of plasticity:

$$
P=\sigma_{y} \frac{2}{3}\left(\frac{\varphi}{3 Z f_{c}}\right)^{1 / 4} J\left(\left(\frac{\varphi}{3 Z f_{c}}\right)^{1 / 4} \sqrt{\frac{R_{p}}{h_{0}}}\right)
$$

In order to provide a numerical estimate of the pressure at the onset of friction, we use the following parameters:

$Z=6, f_{\mathrm{c}}=0.6, \phi=3.10^{-2}, R_{p}=2.10^{7} \mathrm{~m}, h_{0}=10^{-10} \mathrm{~m}$.

\section{References}

1. Baklouti, S., T. Chartier, and J.F. Baumard, Mechanical properties of drypressed ceramic green products: The effect of the binder. Journal of the American Ceramic Society, 1997. 80(8): p. 1992-1996.

2. Desfontaines, M., et al., Characterisation of the green machinability of AlN powder compacts. Journal of the European Ceramic Society, 2005. 25(6): p. 781-791. 
3. Baklouti, S., T. Chartier, and J.F. Baumard, Binder distribution in spray-dried alumina agglomerates. Journal of the European Ceramic Society, 1998. 18(14): p. $2117-2121$.

4. Tanaka, S., C.C. Pin, and K. Uematsu, Effect of organic binder segregation on sintered strength of dry-pressed alumina. Journal of the American Ceramic Society, 2006. 89(6): p. 1903-1907.

5. Zainuddin, M.I., S. Tanaka, and K. Uematsu, Effect of Segregation of a Polyacrylic Acid (PAA) Binder on the Green Strength of Dry-Pressed Alumina Compacts. Journal of the American Ceramic Society, 2008. 91(12): p. 38963902.

6. Hondo, T., et al., Influence of binder layer of spray-dried granules on occurrence and evolution of coarse defects in alumina ceramics during sintering. Journal of the European Ceramic Society, 2018. 38(4): p. 1846-1852.

7. Lintingre, E., et al., Control of particle morphology in the spray drying of colloidal suspensions. Soft Matter, 2016. 12(36): p. 7435-7444.

8. He, S., et al., Structure and Mechanical Performance of Poly(vinyl Alcohol) Nanocomposite by Incorporating Graphitic Carbon Nitride Nanosheets. Polymers, 2019. 11(4).

9. Le Bray, Y. and M. Prat, Three-dimensional pore network simulation of drying in capillary porous media. International Journal of Heat and Mass Transfer, 1999. 42(22): p. 4207-4224.

10. Zhang, Y., et al., Effect of poly(vinyl alcohol) adsorption on binder segregation during drying. Journal of the American Ceramic Society, 1996. 79(2): p. 435440. 
11. Pauchard, L., Y. Pomeau, and S. Rica, Deformation of elastic shells. Comptes Rendus De L Academie Des Sciences Serie Ii Fascicule B-Mecanique Physique Astronomie, 1997. 324(7): p. 411-418.

12. E.M., L.L.D.a.L., Theory of Elasticty. 3rd ed.: Butterworth-Heinemann

13. Hemmerle, A., M. Schroeter, and L. Goehring, A cohesive granular material with tunable elasticity. Scientific Reports, 2016. 6.

\section{Figures}

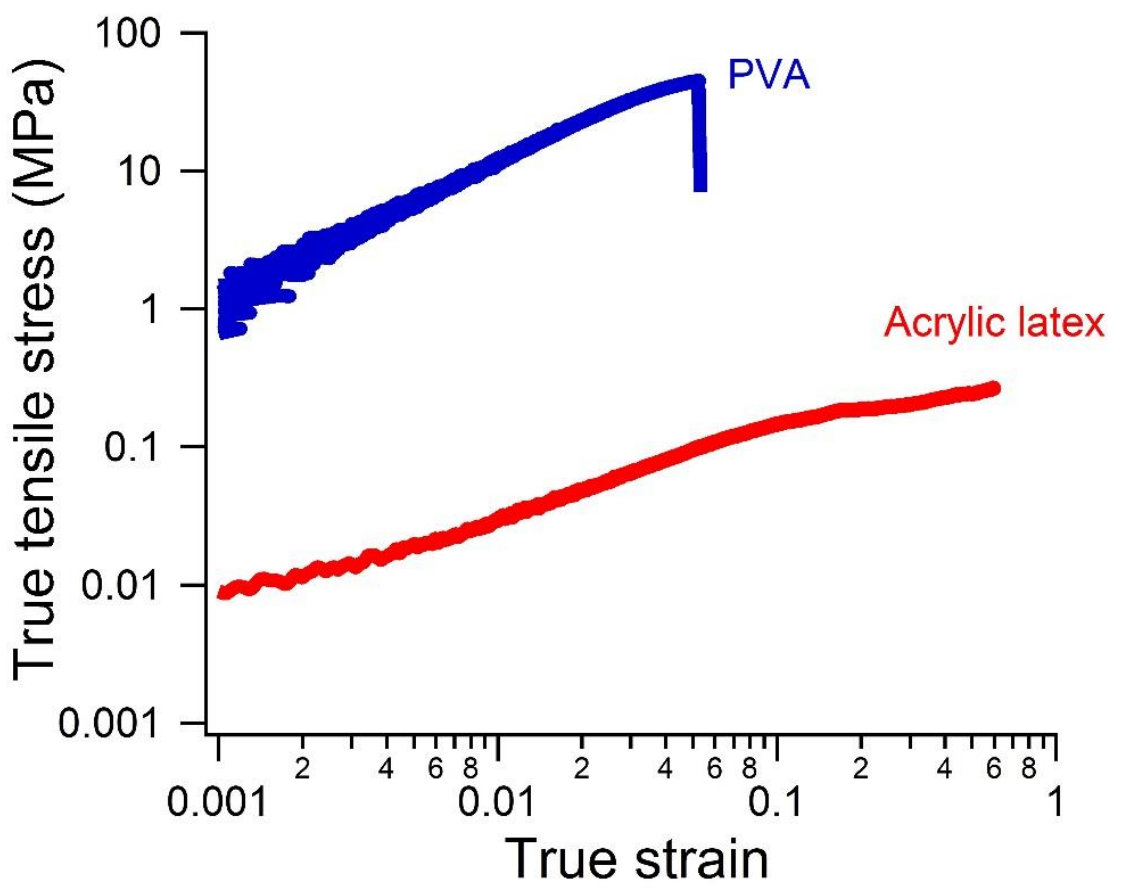

Fig. 1: Tensile

stress as a function of strain measured with the two binders. The slope change in the curve for PVA corresponds to the rupture of the sample. The strain rate was $0.18 \mathrm{~s}^{-1}$ for acrylic latex and $0.36 \mathrm{~s}^{-1}$ for PVA. 


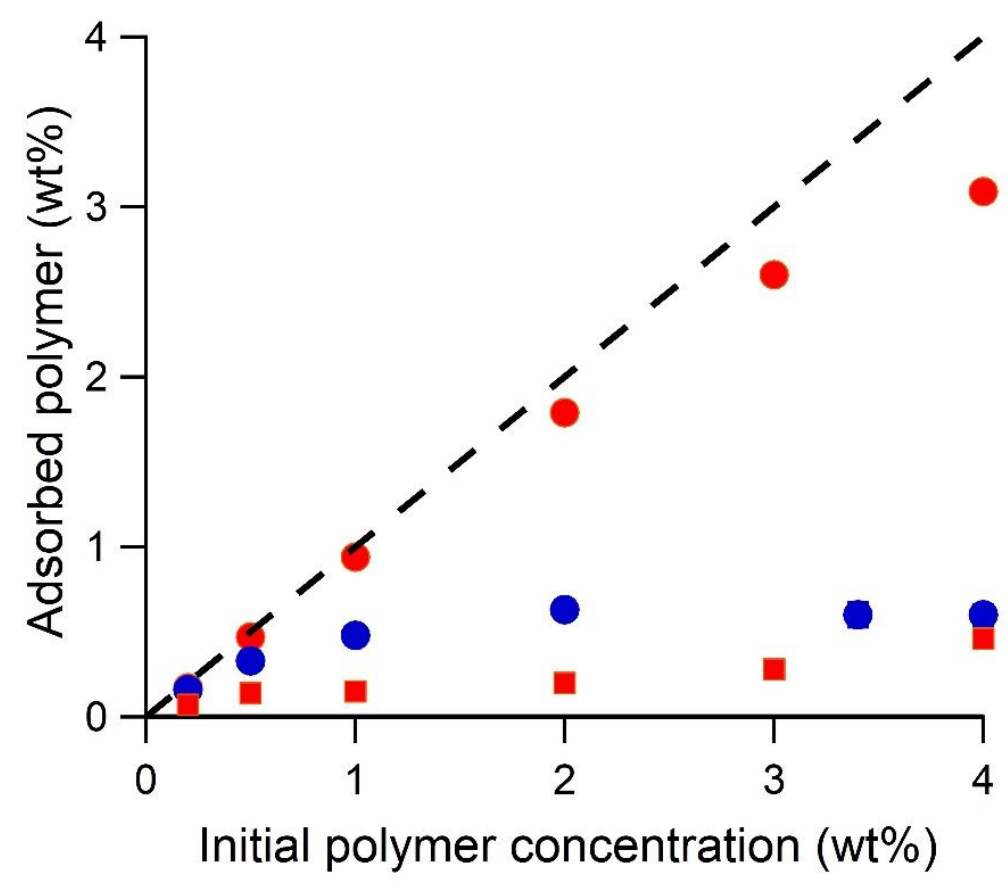

Fig. 2: Quantity of polymer adsorbed on Zirconia as a function of the initial polymer concentration for acrylic latex (red/light markers) and PVA (blue/dark markers). Adsorption was measured in two different acrylic latex samples: a dispersant-free (circles) and a sample with dispersant (squares). The dotted line indicates full polymer adsorption.

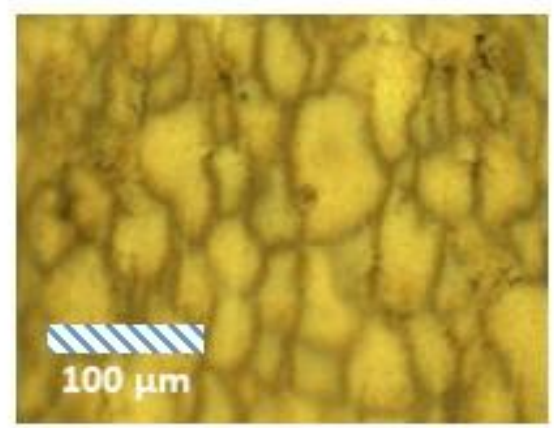

Fig. 3: Optical microscopy image of the fractured surface of a green body after thermal treatment at $300^{\circ} \mathrm{C}$ for $1 \mathrm{~h}$. The spray dried granules were obtained with a Zirconia suspension containing PVA (3.7 wt\% for the dry sample) and dispersant to inhibit 
polymer adsorption on Zirconia. The black parts correspond to the polymer layers degraded by thermal treatment.

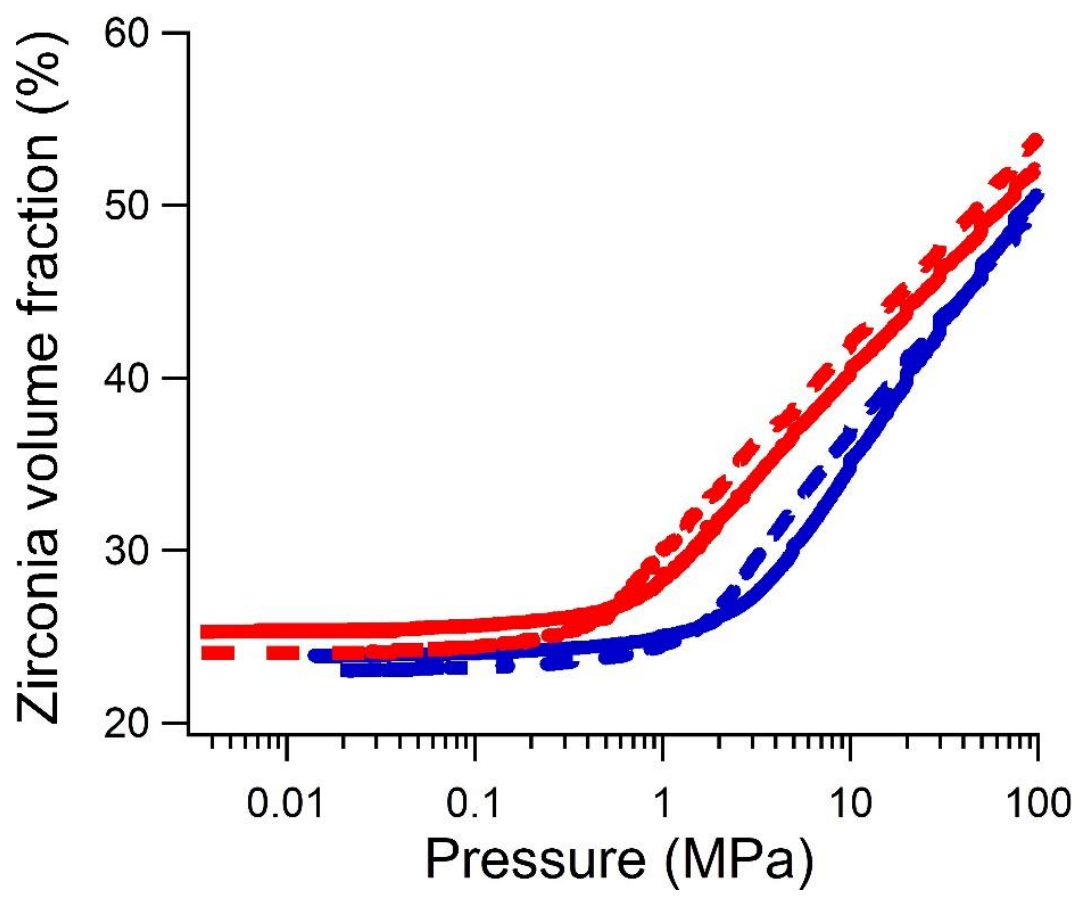

Fig. 4: Compaction curves for dispersant free samples (full lines) and samples containing dispersant (dashed lines) in order to prevent binder (Acrylic latex (red) and PVA (blue)) adsorption and promote the formation of a segregated layer. In all cases, the binder content was $2 \%$ wt. 


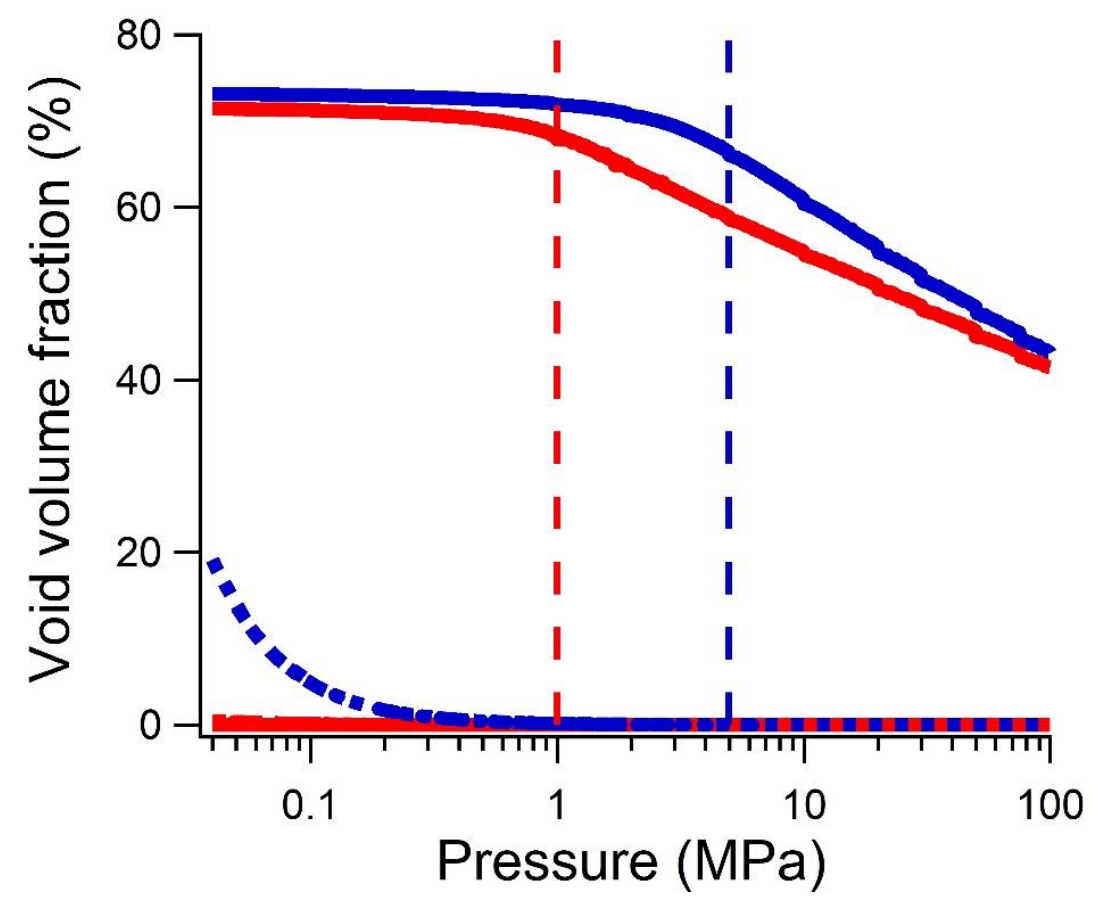

Fig. 5: Void volume fraction as a function of compaction pressure for dispersant-free samples with Acrylic latex (full red line) and PVA (full blue line). The data are the same as in Fig. 4. Additional data corresponds to the computed void volume fraction at vertices filled by the segregated layers of Acrylic latex (red dotted line on the horizontal axis) and PVA (blue dotted line) respectively computed using Eq. (A19) and Eq. (A20) of Appendix A. The dashed vertical lines show the pressure values at which friction between nanoparticles is expected to be at stake respectively in samples with Acrylic latex and PVA. 


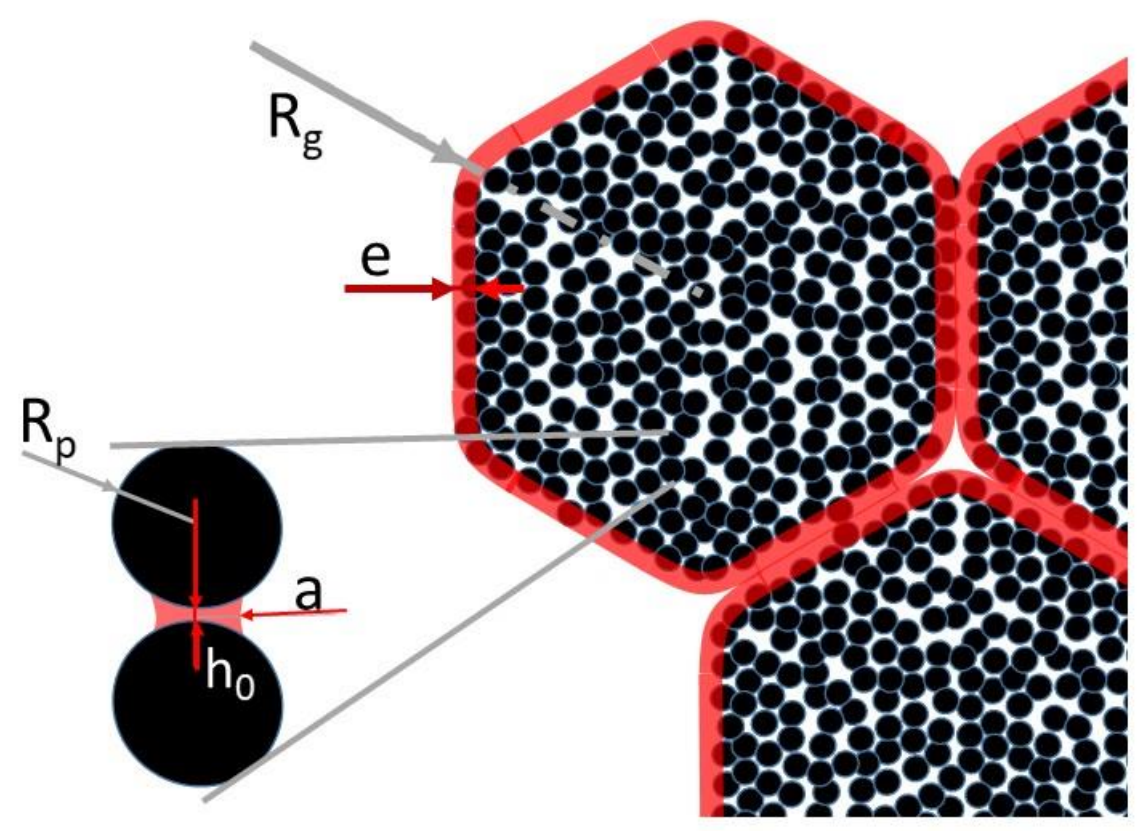

Fig 6: Schematic view of the packing of granules considered in the model in the case of a non-adsorbing polymers. The granules of radius $R_{\mathrm{g}}$ are themselves a packing of nanoparticles of radius $R_{\mathrm{p}}$. The binder is distributed in a shell of thickness $e$ and within the granules, forming bridges between nanoparticles. The height and radius of the bridges are respectively denoted as $h_{0}$ and $a$. 


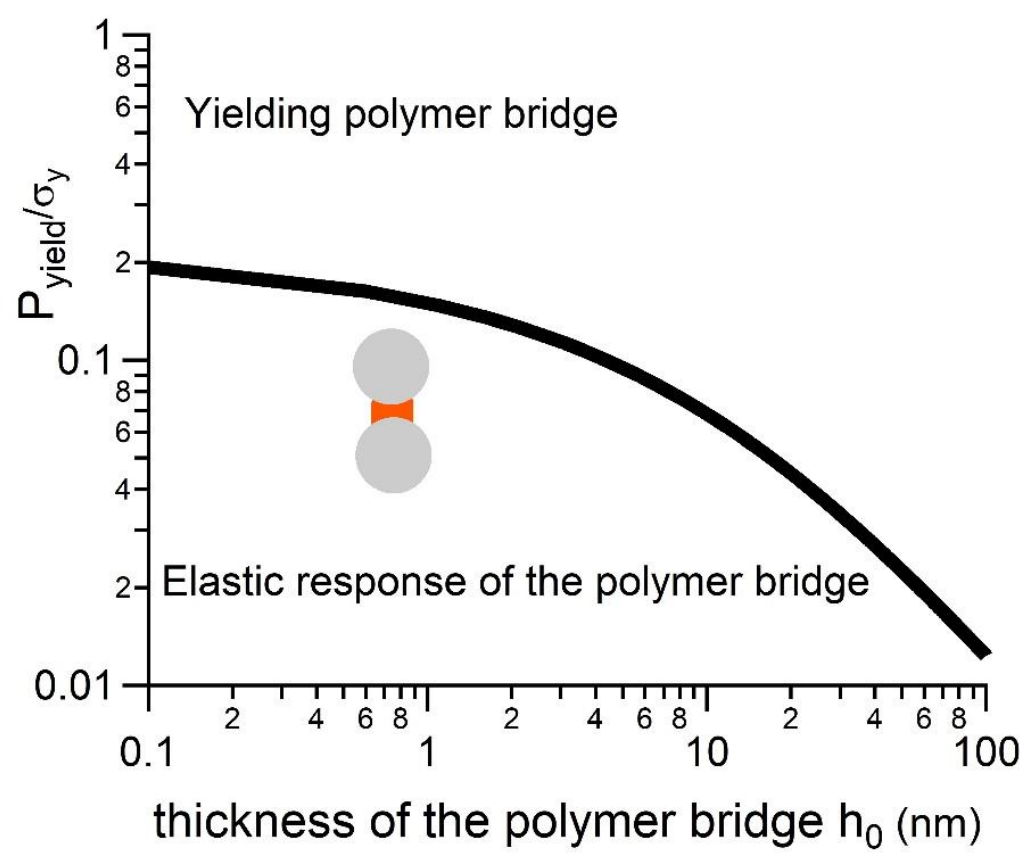

Fig. 7: Yielding pressure for plastic deformation of a polymer bridge between nanoparticles as a function of the thickness of the bridge following eq. (2) and with parameters corresponding to the sample with PVAS as binder. 


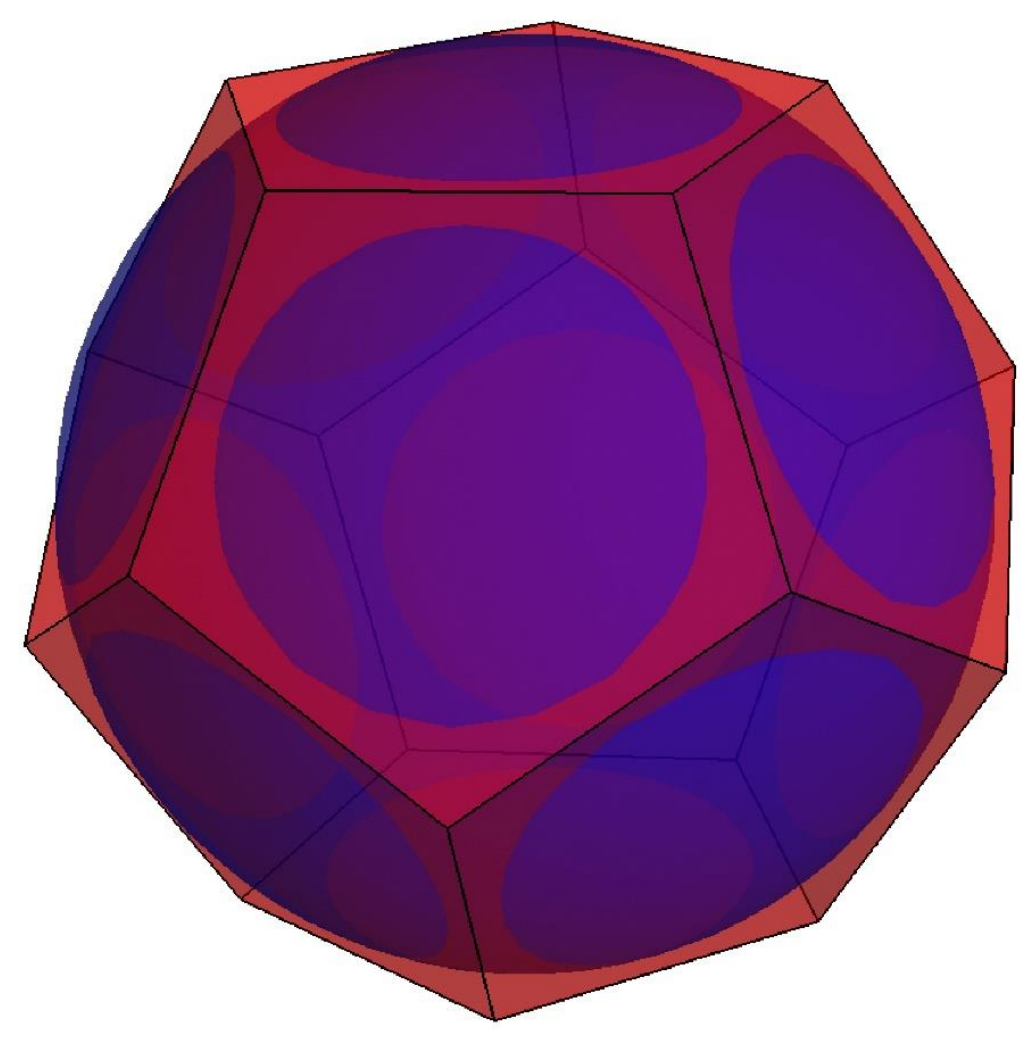

Fig. A.1: The granules under compression progressively transforms into polyhedra. At the end of the process, the voids are mostly on the vertices and on the edges of the polyhedra.

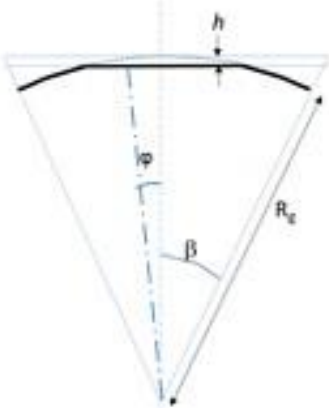


Fig. A.2. Geometry of one face generated by the flattening of the shell one against its neighbours.

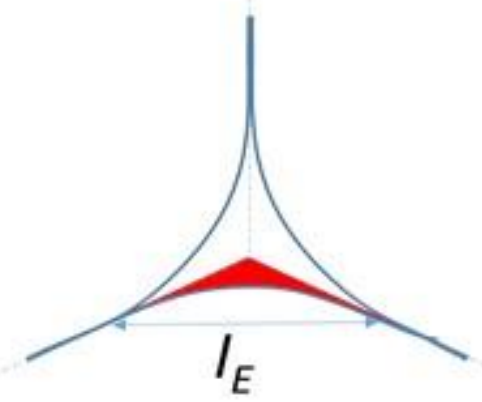

Fig. A3: Shells arrangement at edges. The red part corresponds to the void associated with the granule at the bottom.

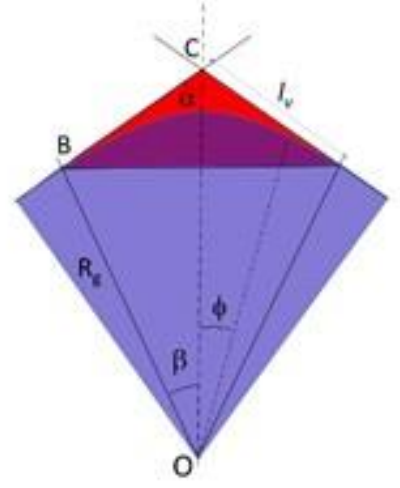

Fig. A.4: Sketch of the granule at a vertex.

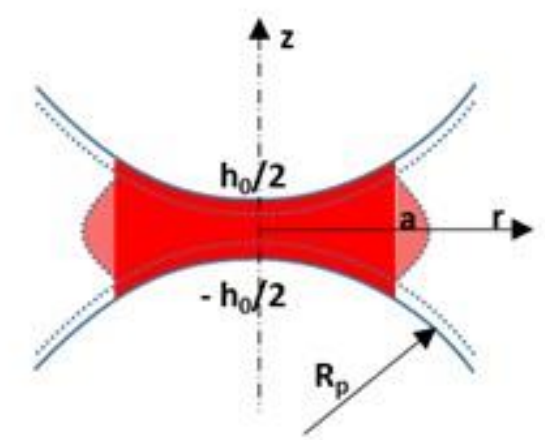

Fig. B1: Scheme of a binder bridge between two nanoparticles. 
